Calderón, F.J.; Cupeiro R.; Peinado A.B.; Lorenzo-Capella, I. (2020) Heart Rate Variability and Exercise, Is There a Physiological Basis? Revista Internacional de Medicina y Ciencias de la $\begin{array}{lllllll}\text { Actividad Física y el Deporte vol. } 20 & \text { (78) pp. 299-320 }\end{array}$ Http://cdeporte.rediris.es/revista/revista78/artvariabilidad1151.htm

DOI: http://doi.org/10.15366/rimcafd2020.78.008

\title{
ORIGINAL
}

\section{VARIABILIDAD DE LA FRECUENCIA CARDIACA Y EJERCICIO. ¿FUNDAMENTACIÓN FISIOLÓGICA?}

\section{HEART RATE VARIABILITY AND EXERCISE, IS THERE A PHYSIOLOGICAL BASIS?}

\author{
Calderón, F.J. ${ }^{1}$; Cupeiro R. ${ }^{2}$; Peinado A.B. ${ }^{3}$ y Lorenzo-Capella, I. ${ }^{4}$ \\ ${ }^{1}$ Profesor Titular. Doctor en Medicina. Departamento de Salud y Rendimiento Humano, Facultad de \\ Ciencias de la Actividad Física y del Deporte (INEF), Universidad Politécnica de Madrid (España) \\ franciscojavier.calderon@upm.es \\ 2 Profesora Ayudante Doctor. Doctora en Ciencias de la Actividad Física y del Deporte. \\ Departamento de Salud y Rendimiento Humano, Facultad de Ciencias de la Actividad Física y del \\ Deporte (INEF), Universidad Politécnica de Madrid (España) rocio.cupeiro@upm.es \\ 3 Profesora Contratado Doctor. Doctora en Ciencias de la Actividad Física y del Deporte. \\ Departamento de Salud y Rendimiento Humano, Facultad de Ciencias de la Actividad Física y del \\ Deporte (INEF), Universidad Politécnica de Madrid (España) anabelen.peinado@upm.es \\ ${ }^{4}$ Profesora Contratado Doctor. Doctora en Ciencias de la Actividad Física y del Deporte. Universidad \\ Camilo José Cela (España) ilcabs@yahoo.es
}

Código UNESCO / UNESCO code: 2411.03 Fisiología humana. Fisiología cardiovascular/ Human Physiology. Cardio-vascular physiology

Clasificación del Consejo de Europa/ Council of Europe classification: 6 . Fisiología del ejercicio/ Exercise Physiology.

Recibido 28 de junio de 2018 Received June 28, 2018

Aceptado 7 de marzo de 2019 Accepted Marcha 7, 2019

\section{RESUMEN}

Este trabajo pretende dos objetivos: 1ํ) dar una visión pedagógica de la complejidad relativa al tratamiento matemático de la variabilidad de la frecuencia cardiaca y $2^{\circ}$ ) analizar si el tratamiento matemático de la señal RR (distancia entre dos ondas $R$ del electrocardiograma) tiene una base fisiológica.

Se revisan los mecanismos fisiológicos que explican uno de los fenómenos de base para el análisis de la variabilidad: la arritmia sinusal respiratoria. Se analizan las 
bases matemáticas, así como los métodos matemáticos de valoración, de la variabilidad. Finalmente, se realiza una revisión del significado fisiológico de las bandas de frecuencia obtenidas mediante las diversas metodologías del tratamiento de la señal RR.

No está claro si los métodos matemáticos de tratamiento de la señal RR pueden ser una herramienta de valoración de la función vegetativa. Por ello, debemos tener precaución al interpretar esta variable, sobre todo en el contexto del ejercicio físico y entrenamiento.

PALABRAS CLAVE: ECG, Frecuencia Cardiaca, Entrenamiento Físico, Actividad Física.

\section{ABSTRACT}

This work has two objectives: 1) to give a pedagogical view about the complexity of the mathematical treatment of heart rate variability and 2) to analyze whether the mathematical treatment of the RR signal (distance between two R waves shown in an electrocardiogram) has a physiological basis.

We reviewed the physiological mechanisms that explain one of the basic phenomena for the analysis of heart rate variability: respiratory sinus arrhythmia. The elementary mathematical bases as well as the mathematical methods of assessing the variability are analyzed. Finally, we offer a critical review of the physiological significance of the frequency bands obtained by the various methodologies of the RR signal processing.

It is not clear whether the mathematical methods of $R R$ signal processing can be an assessment tool for vegetative function. Therefore, this variable must be interpreted with caution, especially in the context of physical exercise and training.

KEY WORDS: ECG, Heart Rate, Physical Training, Physical Activity.

\section{INTRODUCCIÓN}

Desde que se publicó el primer artículo sobre variabilidad de la frecuencia cardiaca (Heart Rate Variability = HRV) (Malmo et al., 1948), el interés por esta metodología ha experimentado un aumento potencial del número de artículos (figura 1). Así mismo, en la base de datos Pubmed existen más de 20 términos en los que el primero es HRV. Los avances tecnológicos permiten realizar cálculos matemáticos complejos en unos pocos minutos y el trabajo en equipo de matemáticos, físicos y fisiólogos son las razones de este aumento en la publicación de trabajos 


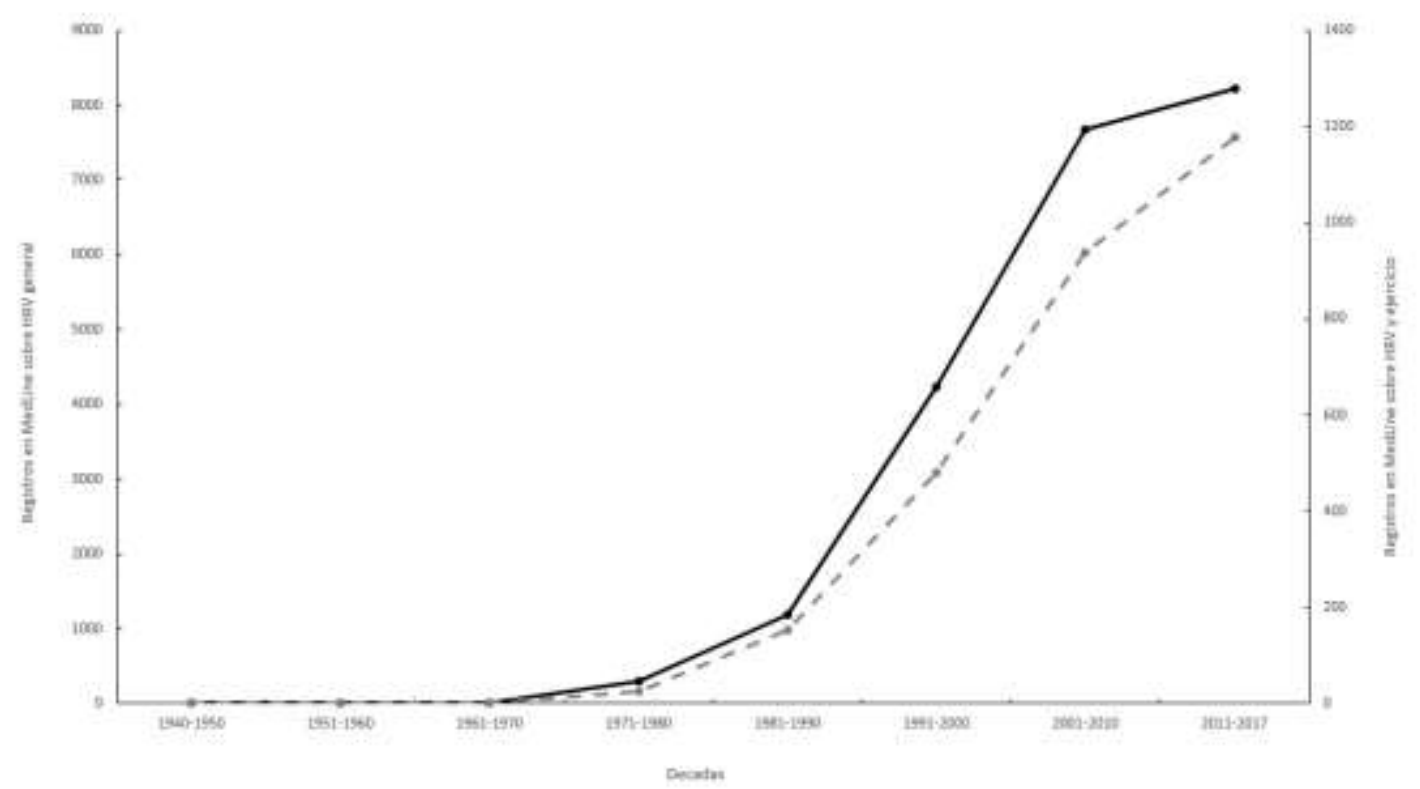

Figura 1. Número de artículos publicados sobre HRV de forma general (línea continua) y en relación con el ejercicio (línea discontinua) tras la búsqueda realizada en la base de datos PubMed (elaboración propia).

Como la actividad cardiaca es una función que se repite en el tiempo, puede ser tratada mediante diferentes metodologías de cálculo. Ésta periodicidad se puede explicar desde el punto de vista fisiológico. Con esta "perspectiva conjunta", el estudio de la HRV ha tenido diversos objetivos. De forma general, el interés de los investigadores ha sido estudiar el equilibrio o balance vegetativo tanto desde una perspectiva fisiológica (C. Silva, Pereira, Cardoso, Moore, \& Nakamura, 2014; V. P. Silva, Oliveira, Silveira, Mello, \& Deslandes, 2015) como patológica. Desde el punto de vista fisiológico, el interés de los investigadores por el estudio de la HRV ha sido para utilizarlo como control de la fatiga o sobreentrenamiento por modificación del balance vegetativo, para caracterizar el proceso de recuperación cardiaca (Mourot et al., 2004) y la bradicardia ocasionada por el entrenamiento (Borresen \& Lambert, 2008; GR Sandercock, Bromley, \& Brodie, 2005).

Sin embargo, esta "alianza" de ciencias tan dispares no ha resultado tan positiva como a priori pudiera parecer. Cuando se ha intentado hacer corresponder los diferentes índices matemáticos, resultado del análisis de la HRV, con la actividad vegetativa sobre el corazón, la confusión reinante es considerable. Se asume que los diferentes índices aportados por las matemáticas se corresponden con la diferente actividad vegetativa (simpática y parasimpática). Esto naturalmente es un craso error, pues no está firmemente demostrado que exista una relación entre los diferentes índices propuestos y la actividad vegetativa de las dos clásicas divisiones del sistema nervioso vegetativo (Casadei, Cochrane, Johnsoton, Conway, \& Sleight, 1995).

En razón a lo expresado anteriormente, el objetivo de este trabajo de revisión es intentar valorar el significado fisiológico de una "herramienta" de medición muy 
utilizada (figura 1) de la actividad vegetativa sobre el corazón. Dada la complejidad de los diferentes métodos matemáticos para valorar la HRV, el trabajo se sustenta principalmente en la parte fisiológica. No obstante, aunque la formación matemática para comprender los diferentes métodos es limitada, se intentará explicar de la forma más pedagógica posible.

\section{MATERIAL Y MÉTODOS}

Con objeto de responder a los objetivos del trabajo, se ha realizado una revisión bibliográfica en la que se incluyeron todos aquellos artículos científicos sobre variabilidad de la frecuencia cardiaca durante el ejercicio. La búsqueda bibliográfica se realizó en las bases de datos Web of Science y PubMed, abarcando el periodo de tiempo desde 1940 hasta 2017. Los términos empleados fueron "heart rate variability", "HRV", "LF/HF" y "autonomic control of heart rate", en combinación con "exercise" y "training".

Un total de 586 resultados respondieron a esta búsqueda. De este total, se descartaron aquellos escritos en idiomas diferentes al castellano, inglés o alemán. A continuación, y mediante la lectura del resumen, se seleccionaron aquellos trabajos que trataban aspectos fisiológicos de la variabilidad de la frecuencia cardiaca.

De los 136 seleccionados tras estos dos primeros pasos, se descartaron también todos los trabajos que fuesen presentaciones en congresos, o no fuese posible encontrar texto completo.

Este proceso de selección dio un resultado final de 43 artículos de investigación, que han sido los utilizados para la presente revisión.

\section{FUNDAMENTOS FISIOLÓGICOS DE LA VARIABILIDAD DE LA FRECUENCIA CARDIACA}

Como muchos órganos, el corazón posee una doble inervación del sistema nervioso vegetativo (SNV). La figura 2 muestra de forma esquemática esta inervación. Además, de los mecanismos reflejos que se pueden establecer, principalmente el barorreflejo, los centros superiores pueden influir sobre éstos 


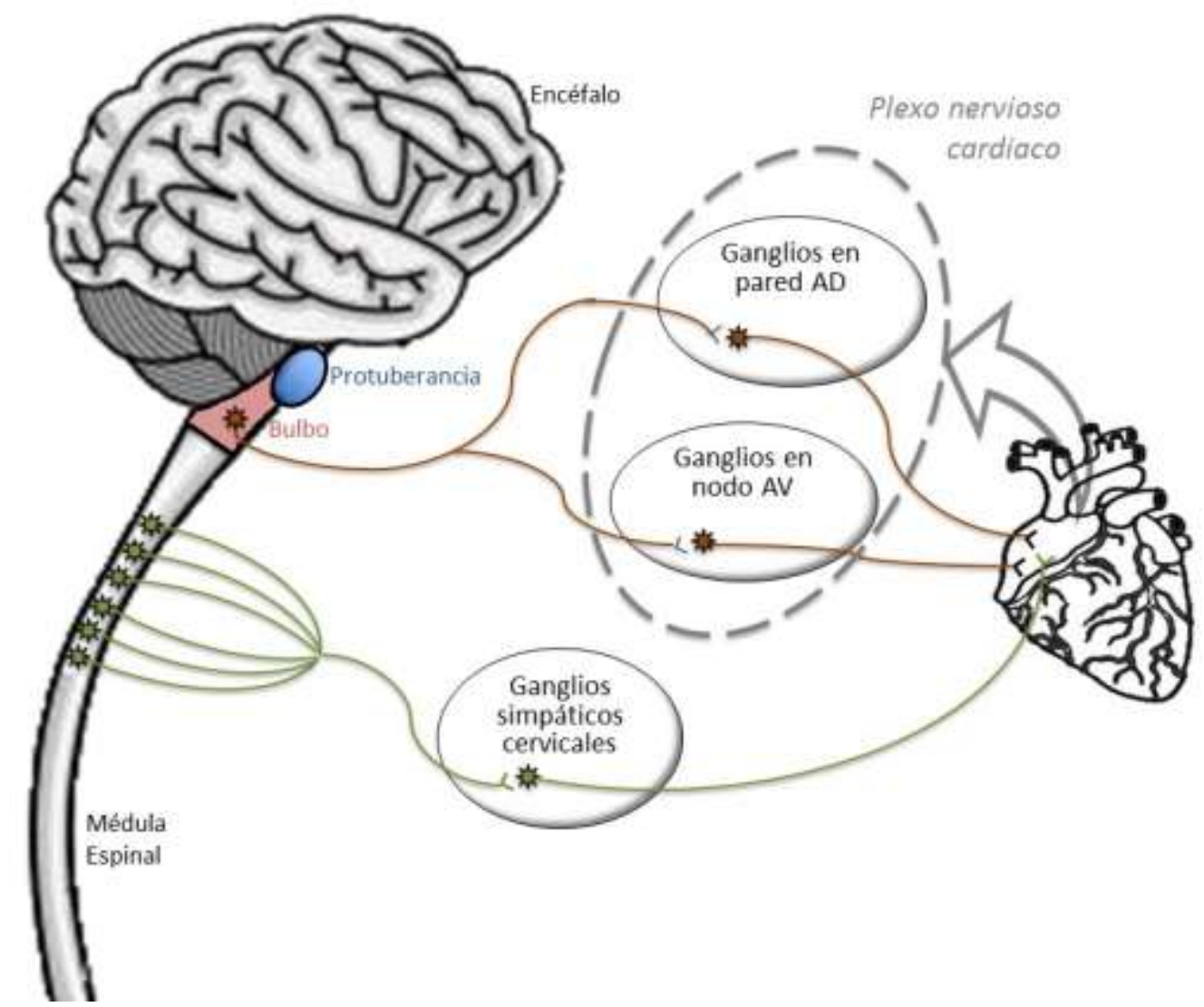

Figura 2. Representación esquemática de la inervación vegetativa del corazón (elaboración propia).

Existen tres zonas del sistema nervioso central relacionadas con la actividad vegetativa sobre el corazón.

1) Se ha demostrado que las neuronas del asta intermedio lateral tienen cierta actividad espontánea (Billman, 2015).

2) El bulbo raquídeo, por su estrecha relación entre los centros para el control respiratorio y cardiovascular (Schramm, 2006).

3) El hipotálamo ejerce control sobre el sistema cardiovascular (Cherniack, Adams, Prabhakar, Haxhiu, \& Mitra, 1989) y que la corteza cerebral puede ejercer efectos sobre la frecuencia cardiaca (Jindal, Gupta, \& Das, 2013; P. Korner, 1971; P. I. Korner, 1971).

Todas estas estructuras se encuentran relacionadas tanto en el sentido vertical como horizontal. La actividad tónica vegetativa probablemente nace en múltiples lugares, considerados en sentido longitudinal, si bien se piensa en la actualidad que podrían existir "marcapasos" centrales de difícil localización y que de forma incorrecta se denominan en singular como comando central (Cherniack et al., 1989; Verberne \& Owens, 1998). La descarga de estos marcapasos se ve influenciada 
por: la actividad de los barorreceptores, mecanismos respiratorios y condiciones locales en el SNC.

Para el objeto de éste trabajo de revisión, la actividad vegetativa sobre el corazón se centra en la frecuencia cardiaca (FC). Ésta es el resultado de la actividad eléctrica de las células marcapasos situadas en los nodos sino-auricular y aurículoventricular. Dicha actividad eléctrica es modulada por las terminaciones nerviosas que llegan a los dos nódulos. Aunque es cierto que el mayor número de células de despolarización lenta se encuentran en el nodo SA, esta función también la ejerce el nodo A-V. Por consiguiente, la acción del sistema vegetativo sobre la FC se realiza en función de la acción de éste en ambos nodos.

El trabajo pionero de Rosenbluth y Simeone (Turner, 1991) da idea de la complejidad de la función vegetativa sobre el corazón. Estos autores estudiaron la respuesta cardiaca a la estimulación aislada (simpática o parasimpática) y a la acción combinada de ambas. Es de resaltar que, aunque se han descrito diferencias de inervación por los dos nervios vagos, los efectos de la estimulación parasimpática se ponen de manifiesto en mayor cuantía cuando se estimulan las terminaciones del simpático. El mecanismo que explica en parte esta interacción es la tendencia de la acetilcolina o de los nucleótidos a disminuir la cantidad de noradrenalina liberada por las terminaciones simpáticas o interaccionar en la membrana de las células marcapasos (Rosenblueth \& Simeone, 1934). En reposo hay un predominio vagal dado que el descenso de la actividad con atropina desencadena taquicardia, mientras el descenso de la actividad simpática provoca un menor descenso de la frecuencia cardiaca (Boron \& Boulpaep, 2012).

Por otra parte, el origen del estudio de la HRV estuvo en determinar los mecanismos fisiológicos que permitieran explicar una manifestación electrocardiográfica relativamente frecuente: la arritmia sinusal respiratoria (ASR). Esta representa las variaciones del ritmo cardíaco por efecto de la respiración y ha venido siendo estudiada desde el siglo XIX (Das, 1989). Se observó que la frecuencia cardiaca fluctúa con los movimientos respiratorios (Coleridge \& Coleridge, 1986; Ludwig, 1847). En otras palabras, el sistema cardiovascular funciona en "sincronía" con el aparato respiratorio. De forma simple y resumida, la FC aumenta con la inspiración y desciende durante la espiración, pero la relación exacta entre la frecuencia cardiaca y las fases del ciclo respiratorio depende de la frecuencia respiratoria y del volumen corriente (Katona \& Jih, 1975) (Jewett, 1964). Seis teorías han sido propuestas para explicar el desarrollo de la arritmia sinusal respiratoria (Kunze, 1972):

1) reflejos originados en el aparato respiratorio: La retroalimentación vagal con origen en los receptores de estiramiento pulmonar es determinante y necesaria para la activación y mediación neural de la $A S R$ en condiciones normales y a elevadas intensidades de ejercicio. Otros mecanismos reflejos, originados en la musculatura respiratoria podrían contribuir a la coordinación cardio-respiratoria. 
2) mecanismo central. En mamíferos, se aceptan dos mecanismos principales que generan la ASR: modulación cardiaca directa de las neuronas vagales preganglionales por impulsos centrales respiratorios e inhibición de la actividad eferente vagal cardiaca durante la inspiración pulmonar.

3) reflejo originado en receptores de la aurícula derecha.

4) mecanismo local que involucra al nodo sinusal.

5) reflejo originado en los barorreceptores y quimiorreceptores. Las fibras eferentes de origen vagal son más poderosamente excitadas durante la expiración por estimulación de quimiorreceptores y el barorreceptors arteriales.

6) oscilaciones de los parámetros de $\mathrm{PpCO} 2$ y pH arterial.

Probablemente, la ASR sea el resultado de la acción de todos ellos. No obstante, y con independencia de los mecanismos, las características de la respiración afectan al control de la frecuencia cardiaca. La amplitud de la ASR es más elevada a 5-6 resp/min y disminuye de forma progresiva cuando aumenta la frecuencia respiratoria. Los trabajos iniciales demostraron que la vagotonía cervical abolía la ASR, aportando evidencias indirectas de su origen nervioso y en contra de la hipótesis de que se trataba de modificaciones vegetativas del corazón provocadas por la expansión del pulmón. Parece que la inervación simpática no es esencial en el desarrollo de la arritmia, aunque no se excluye su participación (Coleridge \& Coleridge, 1986; Katona \& Jih, 1975) . Con posterioridad y mediante el análisis matemático de la HRV se piensa que la ASR pudiera desempeñar un papel fisiológico activo, ya que la sincronización de la ventilación alveolar y la FC en cada ciclo respiratorio, podía suponer en cierta forma un ahorro energético al "suprimir" latidos innecesarios durante la expiración y "evitar" una ventilación ineficaz. (Katona \& Jih, 1975)

\section{LOS MÉTODOS DE ANÁLISIS DE LA VARIABILIDAD DE LA FRECUENCIA CARDIACA}

El tiempo que hay entre dos ondas $\mathrm{R}$, incluso en reposo, no siempre es el mismo, es decir, "varía" en el tiempo. De forma elemental pues, la variabilidad de la frecuencia cardiaca consiste en la "variación" experimentada por la duración en tiempo de dos o más latidos cardiacos. El problema surge cuando se pretende analizar matemáticamente cómo varía el intervalo entre dos ondas $\mathrm{R}$ a lo largo de un determinado tiempo.

\section{Matemáticas elementales y la HRV}

La figura 3 muestra los datos completos durante 120 min de un registro llevado a cabo en nuestro laboratorio (Laboratorio de Fisiología del Esfuerzo, Universidad 
Politécnica de Madrid) con ECG (Jaeger Oxycon Pro, Erich Jaeger, Viasys Healthcare, Germany). Al objeto de ilustrar mejor el comportamiento de la RR, en la figura 4 se representa los datos parciales de 1,5 minutos.

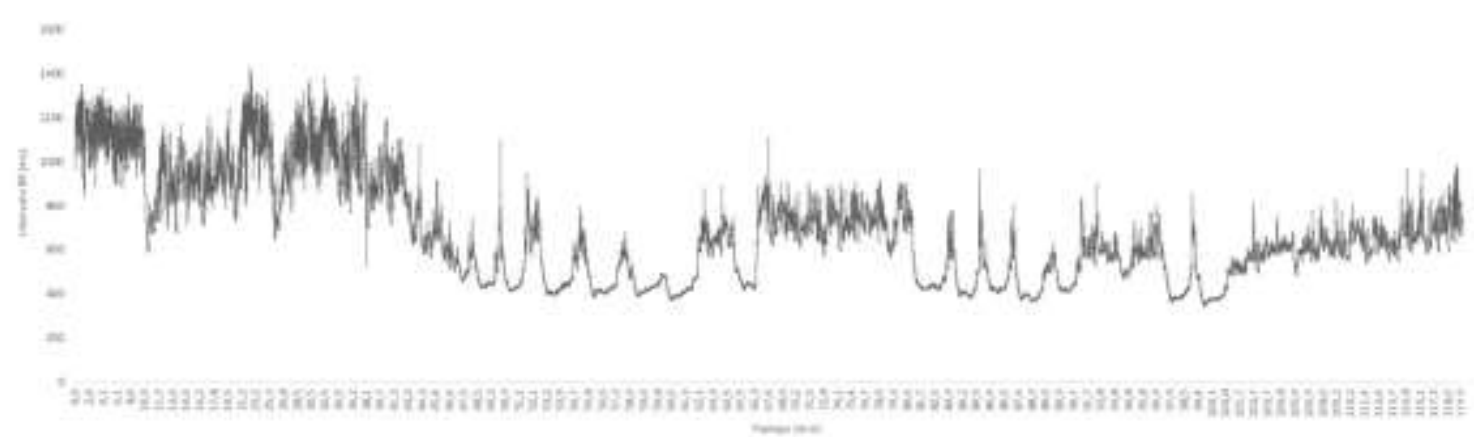

Figura 3. Datos de un registro de frecuencia cardiaca durante 120 minutos.

Es fácil observar dos hechos: $1^{\circ}$ ) que los intervalos RR "varían" a lo largo del tiempo; y $2^{\circ}$ ) que el comportamiento de la función RR/tiempo se puede asemejarse a una función seno.

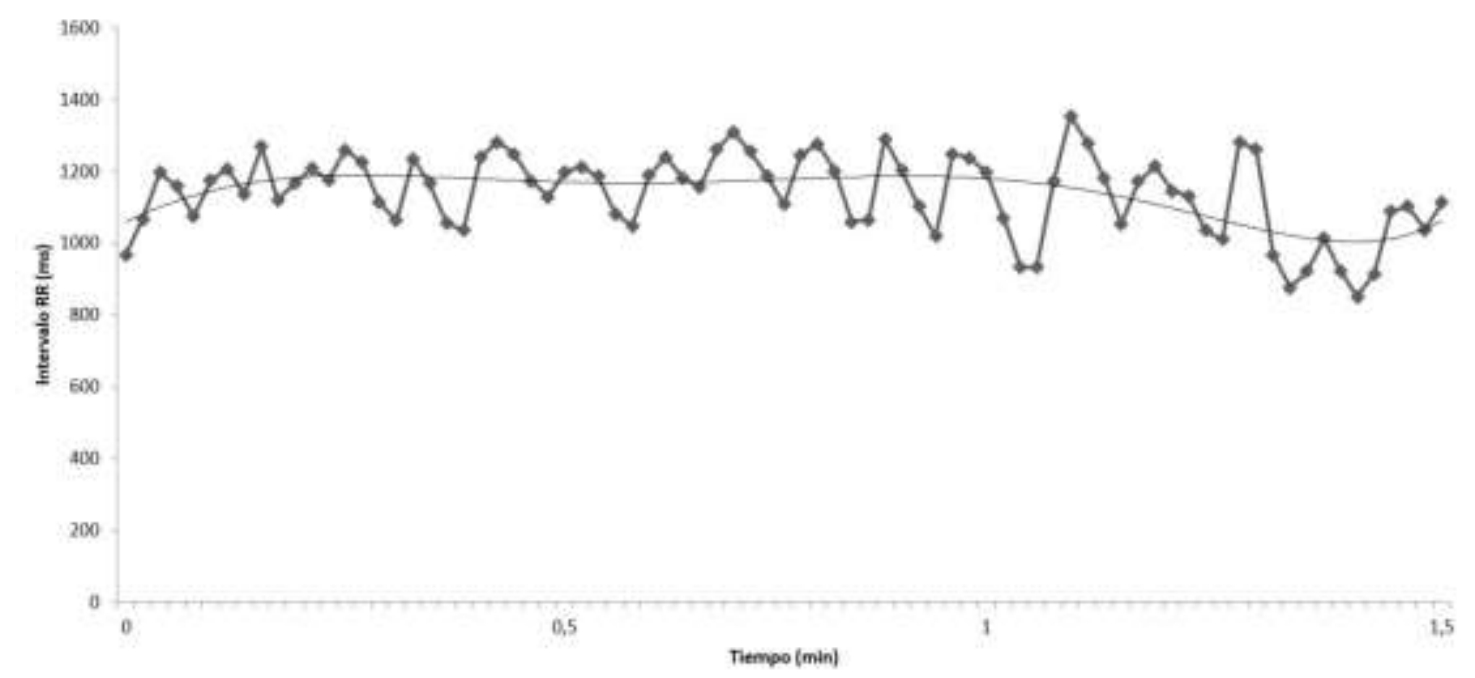

Figura 4. Datos de un registro de frecuencia cardiaca durante 1,5 minutos (elaboración propia).

Al objeto de hacerlo más comprensible, del registro de 1,5 min, se cogen los datos únicamente del minuto a los 1,5 minutos (figura 5), observándose más claramente que los datos se asemejan a una función seno, dando el mayor ajuste polinómico a la serie de datos que permite un programa de cálculo elemental como es el Excel. Esta función dependiente del tiempo $(R R=f(t)$ ajustada a la función seno, igualmente, utilizando otros datos, pudiera ajustarse a una función coseno. 


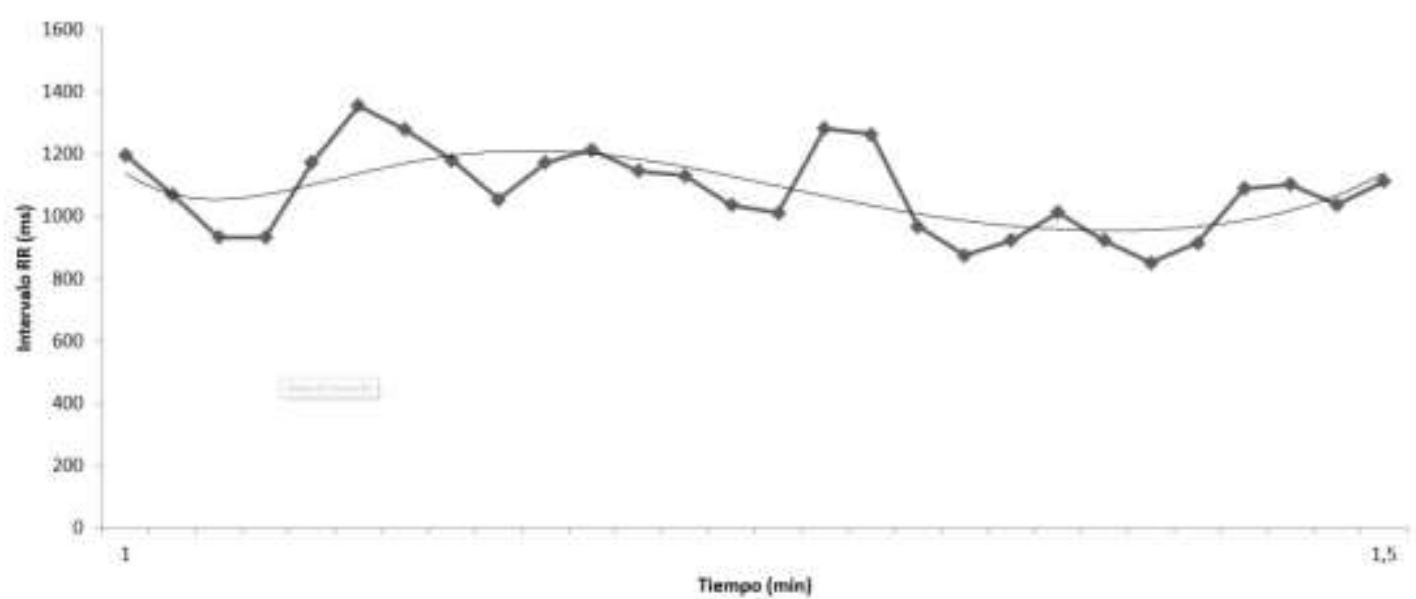

Figura 5. Datos de un registro de frecuencia cardiaca durante 30 segundos (del minuto 1 al minuto 1,5) (elaboración propia).

En definitiva, la realidad es que una sola función no es capaz de "ajustarse" a la serie de datos, de manera que lo que se hace es "buscar" las funciones que mejor se ajustan, normalmente: una combinación de ambas (senos y cosenos). Finalmente, señalar las dificultades que se pueden encontrar cuando se analiza una determinada función RR. Imaginemos que en el registro de la figura 5 se ha podido reconstruir las funciones que mejor representa al registro de los datos RR durante 1,5 minutos y que por las razones que sean en la recogida de datos ha habido "algunas pérdidas". El resultado es que ahora ya no podemos reconstruir la señal original. A la frecuencia con la que se deben obtener datos para poder reconstruir con garantías la señal se denomina frecuencia de Nyquist y debe de ser como mínimo el doble de la frecuencia más alta que representará la señal.

Conocido que la función $\mathrm{RR}$ se puede descomponer en funciones senos y cosenos, el siguiente paso es conocer los valores correspondientes a las frecuencias. Esto se logra gracias al teorema de Fourier, que establece que cualquier función se puede descomponer en el producto matemático de dos o más funciones $(f(x)=$ sen $x$ y $f(x)=\operatorname{cosen} x)$ que representan todos los datos, de manera que la señal total sería correspondiente a un espectro de frecuencias. ¡El producto de las funciones que determinan la función RR es lo que se conoce con el término potencia (PSD = $\mathrm{ms}^{2}$ ), fundamental a la hora de comprender la información que nos aporta el análisis de la HRV!. Las amplitudes de las diferentes funciones senos y cosenos se conocen como coeficientes de Fourier. El cálculo de los coeficientes de Fourier es muy laborioso, pero la capacidad de los ordenadores ha permitido desarrollar diferentes herramientas como la transformación Integral de Fourier, mucho más simple, mediante algoritmos matemáticos: Transformada Rápida de Fourier o la Transformada Discreta de Fourier. Es más, existen programas para el tratamiento de la señal RR que incorporan herramientas de programas complejos como es el Matlab. La ventaja de estos procedimientos es que se puede reconstruir la forma de la función a partir de los coeficientes de frecuencia. 


\section{Métodos matemáticos de valoración de la HRV}

Los diferentes procedimientos matemáticos se basan en la consideración que se puede tener de la señal: estacionaria o no estacionaria. Es decir, considerar que la función $R R=f(t)$ sigue un comportamiento lineal o aleatorio. Sin embargo, como normalmente el registro de la señal $R R$ es de duración finita (en el ejemplo de la figura 3 es de 120 minutos) no existe relación entre la señal obtenida y el resultado matemático de su tratamiento (Transformada Rápida de Fourier o la Transformada Discreta de Fourier), de modo que los matemáticos buscan una solución consistente en formar una nueva sucesión de valores, pero de duración finita. A ese procedimiento lo denominan "ventaneo", porque permite observar la secuencia RR $=f(t)$ parcialmente. No obstante, la desventaja del método es que jno existe una "ventana idónea" en general sino que depende de cada serie individual! En la tabla 1 se muestran los métodos principales aplicados a la valoración de la HRV

La mayor parte de los estudios de HRV y ejercicio se basan en el tratamiento de la señal desde metodologías lineales (señales estacionarias), tales como son los métodos basados en el dominio tiempo (métodos estadísticos y métodos geométricos) y las metodologías del dominio frecuencia (transformadas de Fourier). Tanto si el organismo está en reposo como si se le somete al estrés del ejercicio, el comportamiento temporal de la HRV no se ajusta a una señal estacionaria, de manera que se hace necesario el tratamiento matemático con metodologías no lineales (Tabla 1).

Tabla 1. Resumen de los principales métodos matemáticos de análisis espectral de los intervalos RR

Métodos lineales

1. En el dominio del tiempo
a. Estadísticos
b. Geométricos

2. En el dominio de la frecuencia: Transformada rápida de Fourier (FFT) y Modelo Auto regresivo (AR)

3. En el en el dominio tiempo-escala: transformadas Wavelets.

Métodos no lineales

1. Función de correlación

2. Mapas de retorno 


\section{Reconstrucción del espacio de fase}

- Diagrama de Poincaré: SD1 (Eje transversal): Representa la desviación estándar de la variación instantánea de los intervalos RR. Se aprecia como el diámetro menor de la elipse. Indica la aleatoriedad funcional del sistema y mide la influencia parasimpática sobre el sistema.

- SD2 (Eje longitudinal o línea de identidad): Representa la desviación estándar de la variación continua de los intervalos RR. Se aprecia como el diámetro mayor de la elipse. Indica la dispersión global de los valores de la serie.

- SD1n: Se obtiene al dividir el valor de SD1 por la media de los intervalos RR y luego multiplicar el resultado por 1000. Representa una medición de la actividad vagal.

Otros métodos: bio espectro y diagnóstico del caos

Lo más intuitivo y sencillo en cualquier serie de datos RR es calcular datos estadísticos, tales como la media y desviación estándar. Los datos correspondientes a las figuras 3,4 y 5 indican una considerable variación de los valores RR: $679 \pm 273$ (figura 3), 1140 \pm 112 (figura 4) y 1089 \pm 137 (figura 5). Como señalan los expertos en este tema, la señal no es estacionaria pues tanto la media como la desviación no son constantes. A pesar de lo mencionado la Task Force (Taylor et al., 2014), ¡ha realizado una serie de recomendaciones para valorar la HRV en reposo en series largas (24 horas)! (tabla 2)

Tabla 2. Métodos estadísticos y métodos geométricos para la valoración de la HRV

\begin{tabular}{|c|c|}
\hline \multicolumn{2}{|r|}{ Métodos estadísticos } \\
\hline Variable & Significado \\
\hline SDNN (ms) & Desviación estándar de los intervalos RR \\
\hline SDANN (ms) & $\begin{array}{l}\text { Desviación estándar de los valores promedio de los } \\
\text { intervalos RR registrados durante } 5 \text { minutos }\end{array}$ \\
\hline RMSSD (ms) & $\begin{array}{l}\text { Raíz cuadrada de la media de la suma de los cuadrados de } \\
\text { las diferencias entre los valores de cada RR y el adyacente }\end{array}$ \\
\hline SDNN índex (ms) & $\begin{array}{l}\text { Media de las desviaciones estándar de los intervalos RR } \\
\text { para todos los segmentos de } 5 \text { minutos que se hayan } \\
\text { registrado }\end{array}$ \\
\hline SDSD (ms) & $\begin{array}{l}\text { Desviación estándar de las diferencias entre los valores de } \\
\text { los RR adyacentes }\end{array}$ \\
\hline NN50 count & $\begin{array}{l}\text { Número de pares de intervalos RR adyacentes superiores a } \\
50 \text { ms en el conjunto del registro realizado. Tres posible } \\
\text { variaciones se pueden realizar: 1) contabilizar todos los } \\
\text { pares } R R, 2 \text { ) contabilizar solamente los pares en los que el }\end{array}$ \\
\hline
\end{tabular}


Rev.int.med.cienc.act.fís.deporte - vol. 20 - número 78 - ISSN: 1577-0354

\begin{tabular}{|c|c|}
\hline & $\begin{array}{l}\text { primero de estos sea más prolongado y } 3 \text { ) contabilizar } \\
\text { solamente los pares en los que el segundo de estos sea más } \\
\text { prolongado }\end{array}$ \\
\hline pNN50 & $\begin{array}{l}\text { Número de pares de intervalos } R R \text { adyacentes superiores a } \\
50 \text { ms conjunto del registro realizado dividido por el número } \\
\text { total de intervalos } R R\end{array}$ \\
\hline & Métodos geométricos \\
\hline Índice triangular & $\begin{array}{l}\text { Integral de la distribución de densidad (número de todos los } \\
\text { intervalos RR) dividido por el máximo de la distribución de } \\
\text { densidad }\end{array}$ \\
\hline $\begin{array}{l}\text { Interpolación } \\
\text { triangular del } \\
\text { histograma de } \\
\text { intervalos RR }\end{array}$ & $\begin{array}{l}\text { Amplitud de la línea basal de la distribución medida como la } \\
\text { base de un triángulo aproximando la distribución de los } \\
\text { intervalos RR (se utiliza la mínima diferencia al cuadrado } \\
\text { para encontrar el triángulo) }\end{array}$ \\
\hline \multicolumn{2}{|c|}{ Correlaciones entre los métodos estadísticos y geométricos } \\
\hline \multicolumn{2}{|c|}{$\begin{array}{ll}- & \text { SDNN, índice triangular de HRV, TNN con la Potencia total } \\
- & \text { RMSSD, SDSD, NN50 y pNN50, índice diferencial con HF } \\
\text { - } & \text { SDANN se correlaciona con ULF }\end{array}$} \\
\hline
\end{tabular}

La señal RR se interpreta como un ritmo no autónomo resultado del acoplamiento entre un ritmo interno autónomo con origen en el propio músculo cardiaco y regulado por sistema vegetativo y de una o varias perturbaciones externas de carácter periódico que, como en otros sistemas biológicos (ritmos circadianos, respiración, etc.,), provocan diferentes tipos de fenómenos (acoplamiento del sistema y a la perturbación, cuasi-periodicidad, caos, etc,). Es de destacar que unos de los problemas con los que se encuentran los matemáticos a la hora de analizar las señales biológicas en general es lo que denominan "ruido". Los "ruidos" son ciertos patrones asociados a comportamientos complejos que se detectan analizando los espectros de frecuencia. Un ejemplo es cuando la potencia es proporcional al inverso de la frecuencia, $1 / f$, y que parece es relativamente frecuente en biología.

\section{Las bandas de frecuencia}

La importancia de la descripción de las bandas de frecuencia estriba en la relación que se ha descrito con los diferentes componentes de la función vegetativa y otras funciones fisiológicas (véase relación entre índices matemáticos de la valoración HRV y balance vegetativo). Con independencia del método utilizado en los dominios de la frecuencia o del tiempo-escala, se detectan las siguientes bandas de frecuencia, todas expresadas en valores de potencia $\left(\mathrm{ms}^{2}\right)$ :

1) ULF (ultra low frequency), que describe la potencia en un rango inferior a $0,003 \mathrm{~Hz}$. Longitud ciclo $>5$ horas.

2) VLF (very low frequency), que describe la potencia en un rango inferior a 0,4 $\mathrm{Hz}$. Longitud ciclo $>25 \mathrm{~s}$. 
3) LF (low frequency), que describe la potencia en un rango de frecuencia de 0,04-0,15 Hz. Longitud ciclo > $6 \mathrm{~s}$.

4) HF (high frequency), que describe la potencia en el rango de frecuencia de 0,15-0,4 Hz. Longitud ciclo 2,5 a 6,0 s.

5) VHF (very high frequency), que describe la potencia en el rango 0,40-1,00 $\mathrm{Hz}$.

6) TP (total potency) que describe los intervalos RR durante el periodo. Rango de frecuencia aproximado $<0,4 \mathrm{~Hz}(0,00066-0,34 \mathrm{~Hz})$.

La suma de todas las frecuencias de las bandas constituye la potencia total. Así mismo, es frecuente normalizar y determinar los valores relativos respecto a la potencia total de cada una de las bandas. Así tenemos, las LF y HF normalizadas (valor relativo de cada componente de potencia respecto a la potencia total menos el componente VLF) y las relaciones LFnormalizada/(TP-VLF)100, $H F n o r m a l i z a d a /(T P-V L F) 100$ y $L F / H F$. Las unidades normalizadas tienden a minimizar los efectos de los cambios en la potencia total correspondientes a los valores de los componentes LH y HF. Sin embargo, las unidades normalizadas deben ser siempre citadas junto con los valores absolutos de LH y HF y de esta forma poder describir completamente la distribución de potencia en todos los componentes espectrales.

\section{RELACIÓN ENTRE LOS MÉTODOS DE VALORACIÓN DE LA HRV Y LA FUNCIÓN VEGETATIVA}

Cuestiones metodológicas fundamentales a la hora de interpretar los datos aportados por el tratamiento matemático de la señal RR.

Para la valoración de la HRV en reposo, existe un acuerdo en las condiciones mínimas metodológicas que se deben reunir a la hora de efectuar un registro de corta duración (de 3 a 5 minutos) o de larga duración (24 horas), este último realizado mediante Holter.

1) Modelos experimentales de estudio de la relación HRV/funciones fisiológicas. Las preparaciones de corazones aislados perfundidos han sido ampliamente utilizadas para estudiar los cambios de la función cardiaca sin la influencia de factores externos, tales como la acción del sistema nervioso central, las hormonas circulantes o reflejos nerviosos. En animales y seres humanos se puede proceder al aporte de diversos agentes farmacológicos que simulan los factores externos permitiendo al investigador controlar, manipular y valorar los efectos sobre la activad cardiaca. 
2) Control de la situación del animal experimental. En reposo, la respuesta espectral de la HRV está limitada a $0,5 \mathrm{~Hz}$ y esta se puede descomponer para su análisis. El sujeto debe estar preferentemente tumbado supino y, en su defecto, sentado y en el máximo reposo posible. No es recomendable hacer el registro con el sujeto de pie. Ello es debido a los ajustes hemodinámicos que se producen con los cambios de posición. Diferente es si el objetivo del estudio es analizar los cambios en el espectro de frecuencias por cambios de postura.

3) Control de la respiración: efectos de la respiración sobre la HRV. La influencia de la respiración sobre la HRV es considerable, como se ha señalado previamente y han indicado diversos autores (Cardiology, 1996; Francois Cottin, Durbin, \& Papelier, 2004). Se han encontrado diferencias en la potencia del espectro de la frecuencia cardiaca cuando se respira a diferentes frecuencias $(0,15,0,20,0,30$, 0,40 y $0,50 \mathrm{~Hz}$ ); a medida que aumenta la frecuencia respiratoria desciende el componente HF del espectro y aumenta la relación LF/HF. La espiración provoca un aumento de la HRV y una mayor manifestación vagal. Por tanto, se aconseja que la frecuencia respiratoria sea de 0,15 a $0,25 \mathrm{~Hz}$, o bien de 12 a $14 \mathrm{resp} / \mathrm{min}$.

4) Protocolo de medición. Para la correcta valoración de la señal $R R$ se requiere una serie de duración no inferior a 10 veces la longitud de onda de LF ( 6 a 25 segundos), ya que de esta forma se puede tener cierta seguridad de que la señal es estable. Esto supone que el registro tenga una duración no inferior a 2 min, aunque se establece como medida estándar de corta duración los 5 min (1025 puntos) para poder asegurar series estacionarias que describan adecuadamente cada espectro de frecuencia. Es decir, los registros presentados en las figuras 4 y 5 son incorrectos metodológicamente. Si se han realizado es por simplificar la exposición de un tema muy complejo. Por otra parte, y relacionado con el control de la situación del animal experimental, emplear métodos estadísticos durante el ejercicio o postejercicio invalida los resultados.

5) Otros factores que pueden influir: edad, sexo, condiciones ambientales (temperatura, humedad, altitud), volemia, nivel de condición física y herencia. Parece comprobado que existe un predominio del sistema nervioso simpático en los neonatos, pero es discutible el comportamiento que sigue a lo largo de la edad. En general, con la edad va disminuyendo la actividad parasimpática, de manera que disminuye la variabilidad de los intervalos RR (F Cottin, Papelier, \& Escourrou, 1999; C.-D. Kuo et al., 1999; Tanaka et al., 2000). De manera similar, los resultados respecto a la HRV entre los dos sexos son contradictorios. Se ha sugerido un predominio vagal en los varones respecto a las mujeres, para una misma edad ( $T$. B. Kuo et al., 1999), mientras Umetani y col. no observaron diferencias en la HRV en mujeres de 30 años respecto a varones de la misma edad (Evans et al., 2001). La bradicardia como signo de condición física ha sido valorada mediante los valores más elevados de HF y menores de LF. Se han observado valores de HF más elevados y en menor medida de LF (Umetani, Singer, McCraty, \& Atkinson, 1998). Asimismo, la condición cardiorrespiratoria se asocia con la carga genética de las 
características de la HRV (Achten \& Jeukendrup, 2003; Singh, Larson, O'Donnell, \& Levy, 2001; Singh et al., 2002; Singh et al., 1999).

6) Finalmente, hay que tener en cuenta la reproducibilidad individual que presenta esta señal. En ese sentido, para registros de HRV de corta duración, Marks y col. (1999) (39) encontraron coeficientes de correlación ( $r 2$ ) entre 0,86-0,90 para los análisis temporales y de 0,67-0,96 para los análisis espectrales en dos ocasiones espaciadas por una semana entre cada medición encontraron coeficientes de correlación de oscilaban entre $0,76-0,80$ para análisis espectrales en dos ocasiones en las que los registros fueron separadas por dos meses (40).

\section{Relación entre las bandas de frecuencia y fisiología}

Pocos estudios han intentado "medir" simultáneamente la actividad vegetativa y la HRV (Hughson, Sutton, Fitzgerald, \& Jones, 1977; Pagani et al., 1986; Tsuji et al., 1996). Lo que a todas luces deja en evidencia los trabajos que intentan explicar los resultados de la HRV sin haber medido la actividad vegetativa. Desde el punto de vista de la relación HRV y ejercicio, los dos grandes objetivos han sido:

1) Estudio de la HRV en reposo, al objeto de caracterizar el efecto del entrenamiento sobre la frecuencia cardiaca de reposo y si a lo largo de un proceso de entrenamiento se produce alguna modificación que pudiera indicar un correcto 0 incorrecto proceso de adaptación del organismo. En relación a este último aspecto se ha tratado de diagnosticar y/o prevenir un estado de sobre-entrenamiento mediante la modificación del diagrama de Poincare que pudiera reflejar fatiga cardiaca.

2) Estudio de la HRV en ejercicio al objeto de intentar explicar los ajustes cardiorespiratorios al ejercicio de diversas características: incremental, estable, intermitente, dinámico versus estático, etc.

En la tabla 3 figuran los datos de algunos autores que han abordado el estudio de la HRV, con independencia de si es en reposo o durante el ejercicio. Respecto a las ondas de frecuencia habitualmente medidas por los métodos descritos y que fenómeno fisiológico está relacionado se puede concluir, aunque sea de forma simplificada, lo siguiente:

- Espectro de frecuencia HF en relación con la actividad vagal y con el modelo respiratorio adoptado. Además, se ha relacionado con modulación simpática de las resistencias vasculares periféricas.

- Espectro de frecuencia LF, en los inicios de la investigación, se relacionó con la actividad simpática, pues se producían cambios de potencia de este espectro por el sistema renina-angiotensina-aldosterona y otros factores a nivel local. Estos fenómenos fisiológicos se ponían de manifiesto con cambios en la volemia debidos 
a los cambios de postura. Posteriormente, se ha vinculado el espectro LF tanto con la actividad simpática como parasimpática. Además, se ha establecido relación entre la termorregulación y la amplitud de la potencia LF.

- Espectro VLF, similarmente al espectro LF, también se ha relacionado VLF con: sistema renina-angiotensina-aldosterona, termorregulación, volemia, tono vasomotor y actividad tanto simpática como parasimpática.

- Espectro ULF relacionado con algunos ritmos circadianos como la temperatura.

- Relación LF/HF relacionado con el balance o equilibrio vegetativo con la actividad parasimpática, la termorregulación y cambios de la volemia debidos a la postura. Se ha estimado que el cociente debe de estar entre 1,5 y 2,0 o su equivalente porcentual (15-200 \%) para un registro de cinco minutos en posición supina. Valores sobre este rango indicarán un mayor predominio simpático y valores por debajo reflejarán un mayor predominio parasimpático. En reposo y en un sujeto sano, un valor elevado de esta índice resulta un indicador de riesgo, de forma que una disminución significativa de HF podría indicar una seria degradación del sistema vagal.

Tabla 3. Trabajos que han abordado la relación entre las bandas de frecuencia resultado del análisis de la HRV y los mecanismos fisiológicos.

\begin{tabular}{|c|c|c|}
\hline Autor & $\begin{array}{c}\text { Bandas de frecuencia } \\
\text { descritas }\end{array}$ & Relación fisiológica establecida \\
\hline Sayers (1973) & $\begin{array}{l}\text { - Baja frecuencia }(<0,05 \mathrm{~Hz}) \\
\text { - Media frecuencia }(0,06 \mathrm{a} \\
0,10 \mathrm{~Hz}) \\
\text { - Alta frecuencia }(>0,15 \mathrm{~Hz})\end{array}$ & $\begin{array}{l}\text { - Termorregulación } \\
\text { - Control barorreflejo } \\
\text { - Fluctuaciones respiratorias }\end{array}$ \\
\hline Chess y col. (1975) & - Alta frecuencia & - Actividad parasimpática \\
\hline Akselrod y col. (1985) & $\begin{array}{l}\text { - Alta frecuencia }(>0,15 \mathrm{~Hz}) \\
\text { - Baja y media frecuencia } \\
(0,05 \text { a } 0,10)\end{array}$ & $\begin{array}{l}\text { - Actividad parasimpático } \\
\text { - Actividad simpática y el sistema renina- } \\
\text { angiotensina }\end{array}$ \\
\hline Huang y col. (1997) & $\begin{array}{l}\text { - Alta frecuencia (HF: 0,15 a } \\
0.5 \mathrm{~Hz} \text { ), } \\
\text { - Media frecuencia (MF: } 0,08 \\
\text { a } 0,15 \mathrm{~Hz} \text { ) } \\
\text { - Baja frecuencia (LF: } 0,02 \mathrm{a} \\
0,08 \mathrm{~Hz} \text { ). }\end{array}$ & \\
\hline Hedellin y col. (2001) & $\begin{array}{l}\text { - Ultra baja frecuencia } \\
\text { - Baja frecuencia }\end{array}$ & $\begin{array}{l}\text { - Actividad parasimpática } \\
\text { - Modulación simpática de las resistencias } \\
\text { periféricas vasculares. }\end{array}$ \\
\hline Serrador y col. (1999) & - Ultra baja frecuencia & $\begin{array}{l}\text { - Ritmo circadiano de la FC y su posible } \\
\text { relación con el control hipotalámico }\end{array}$ \\
\hline $\begin{array}{l}\text { Bonaduce y col. } \\
\text { (1998) }\end{array}$ & $\begin{array}{l}\text { - Incremento de la actividad } \\
\text { espectral y del VLF }\end{array}$ & $\begin{array}{l}\text { - Sistema renina-angiotensina aldosterona } \\
\text { (inhibición de la ECA); cambios en la volemia y } \\
\text { barorreflejo }\end{array}$ \\
\hline
\end{tabular}


Rev.int.med.cienc.act.fís.deporte - vol. 20 - número 78 - ISSN: 1577-0354

\begin{tabular}{|l|l|l|}
\hline Spinelli y col. (1999) & $\begin{array}{l}\text { - Incremento de la actividad } \\
\text { espectral } \\
\text { - Muy baja frecuencia }\end{array}$ & $\begin{array}{l}\text { - Cambios en la volemia y barorreflejo } \\
\text { - Sistema parasimpático }\end{array}$ \\
\hline Taylor y col. (1998) & $\begin{array}{l}\text { - Potencia espectral } \\
\text { - VLF }\end{array}$ & $\begin{array}{l}\text { - Sistema parasimpático } \\
\text { - Sistema parasimpático-simpático }\end{array}$ \\
\hline Bianchi y col. (1991) & - VLF & - Ritmos circadianos (sueño/vigilia) \\
\hline $\begin{array}{l}\text { Houle y Billman } \\
\text { (1999) }\end{array}$ & - LF & $\begin{array}{l}\text { - Variaciones tanto del sistema simpático como } \\
\text { parasimpático. }\end{array}$ \\
\hline Arai y col. (1989) & - LF & $\begin{array}{l}\text { - Barorreeflejo, termorregulación y ajuste } \\
\text { cardiovascular a diferentes situaciones }\end{array}$ \\
\hline Tulppo y col. (2003) & - Cambios LF/HF & - Balance vegetativo \\
\hline lellamo y col. (2002) & - Cambios LF/HF & - Balance vegetativo \\
\hline $\begin{array}{l}\text { (Pomerantz y col., } \\
\text { 1985); (Hayano y col., } \\
\text { 1996) }\end{array}$ & - HF & - Modelo respiratorio, tono vagal \\
\hline $\begin{array}{l}\text { Hedelin y col. (2000) } \\
\text { Pichot y col., 2000); }\end{array}$ & - HF & - LF/HF \\
\hline Pagani y col. (1986). & & $\begin{array}{l}\text { - Balance vegetativo sobre corazón y } \\
\text { resistencia vasculares periféricas }\end{array}$ \\
\hline Jensen-Urstad (1997) & - LF/HF & - Balance vegetativo \\
\hline Mateo y col. (2001) & - VLF & - Actividad simpática \\
\hline
\end{tabular}

Consideramos que la confusión reinante es cuanto menos dudosa cuando se intenta establecer una relación entre los parámetros medidos en la HRV y los mecanismos fisiológicos. Los investigadores parecen admitir que la HF refleja la actividad vagal, mientras que el componente LF es más controvertido, no sabiendo realmente si es el resultado de una actividad sólo simpática o de ambas divisiones del SNV. Por consiguiente, la relación LF/HF tampoco demuestra una actividad vegetativa concreta en contraposición a lo que señalan algunos investigadores (Piccirillo et al., 2009). Por último, se desconoce el papel fisiológico de los otros dos componentes del espectro (ULF y VLF), que en determinadas ocasiones constituyen más del 90 $\%$ de la potencia total del espectro. Así pues, es problemático explicar el comportamiento de la HRV en los dos campos señalados anteriormente: reposo y ejercicio.

1) Estudio de la HRV en reposo. En reposo, los deportistas muy entrenados con alto componente de resistencia deberían manifestarse por un elevado valor del espectro HF, una disminución no muy pronunciada en el componente LH y de la relación LF/HF. Este comportamiento debería ser el argumento para un predominio del SNV parasimpático que justificara la bradicardia consecutiva al entrenamiento. Sin embargo, los valores de potencia del espectro señalados no han sido firmemente demostrados (Pagani et al., 1986; GRH Sandercock \& Brodie, 2006), entre otros motivos por la mencionada confusión reinante y porque, probablemente, hay muchas diferencias a nivel individual (GR Sandercock et al., 2005). Además, no 
se tiene en cuenta la influencia de los "moduladores" intrínsecos y extrínsecos que actúan modulando la actividad vegetativa:

a. Modulación intrínseca. Ésta se refiere a todos los mecanismos moleculares que se pueden desarrollar en el corazón, preferentemente a nivel de los nodos sinusal y A-V. Como ya señalara casi hace un siglo Rosenbluth (Goldberger, Challapalli, Tung, Parker, \& Kadish, 2001), la interrelación entre las dos divisiones del SNV es muy notable, de manera que en cualquier situación ambas divisiones (simpática y/o parasimpática) actúan de forma contante. Las terminaciones nerviosas parasimpáticas a nivel del nodo sinusal son ricas en acetilcolinesterasa de manera que el efecto de cualquier "pulso vagal" es relativamente breve porque la acetilcolina es hidrolizada. Sin embargo, en reposo, la influencia del vago prevalece probablemente por la reducción de noradrenalina liberada por las terminaciones simpáticas y un descenso de la actividad colinérgica al estímulo adrenérgico.

b. Modulación extrínseca. Los centros nerviosos superiores y los mecanismos reflejos intervienen en el control cardio-respiratorio. Parece que estos mecanismos pudieran funcionar de forma oscilatoria en periodos cortos o largos generando ritmos de descarga neuronal, lo que complica más aun las inferencias que se pueden extraer del análisis de la HRV.

2) Estudio de la HRV en ejercicio. Si confusa es la relación métodos de análisis $\mathrm{HRV} /$ mecanismos fisiológicos en situación de reposo, se acrecienta cuando se estudia el comportamiento de la RR durante el ejercicio. Por ejemplo, conforme se incrementa la FC durante el ejercicio, la banda HF debería perder magnitud en la potencia total. Sin embargo, algunos autores han demostrado un aumento de la HF, en los límites máximos del esfuerzo, atribuida al aumento exagerado de la actividad respiratoria que ejercería un efecto "mecánico" sobre el corazón (Rosenblueth \& Simeone, 1934). Por consiguiente, siguiendo la relación establecida por diferentes investigadores y resumida más arriba, debería descender la HF y aumentar la proporción en la potencia espectral total del componente LF, con el consiguiente incremento de la relación LF/HF. Esto no ha sido demostrado de forma concluyente (Sarmiento Montesdeoca et al., 2009).

Al no corresponder los índices habituales, los investigadores han empleado parámetros más concretos de los espectros de frecuencia. El comportamiento de las variables biológicas está sujeto a la teoría del caos (Lombardi, 2000; GRH Sandercock \& Brodie, 2006), de manera que hay que utilizar métodos ajustados a este tipo de comportamientos. La utilización de métodos inadecuados, por ejemplo estadísticos, para valorar la HRV durante el ejercicio es un grave error (Hagerman, Berglund, Lorin, Nowak, \& Sylvén, 1996). A pesar del esfuerzo de los investigadores los resultados no son nada concluyentes, no pudiéndose 
establecer una relación entre las diferentes bandas de frecuencia y una explicación fisiológica única y coherente (Casadei et al., 1995).

En resumen, a pesar de la cantidad ingente de artículos relacionados con la HRV, es pronto todavía para establecer que los diferentes métodos matemáticos de tratamiento de la señal RR puedan ser una herramienta de valoración y diagnóstico de la función vegetativa en la clínica (Casadei et al., 1995). La complejidad de los análisis de la HRV vienen dados por los siguientes motivos: $1^{\circ}$ ) la complejidad anatomo-funcional de la actividad vegetativa sobre el corazón, preferentemente sobre los nodos sinusal y aurículo-ventricular, $\left.2^{\circ}\right)$ por la actividad los mecanismos centrales y reflejos que pueden regular la actividad vegetativa sobre el corazón, $\left.3^{\circ}\right)$ los efectos que a nivel del nodo sinusal y aurículo-ventricular pueden tener la acetilcolina y catecolaminas y $\left.4^{\circ}\right)$ porque el tratamiento de las señales requiere un dominio de las diferentes técnicas de análisis, que se pueden salvar por una buena relación entre la matemática y la fisiología. Pero si alejados estamos todavía de asumir el estudio de la HRV en la clínica, el problema se acrecienta cuando se aplican las diferentes metodologías a la respuesta y adaptación cardiaca al ejercicio y entrenamiento, respectivamente.

\section{REFERENCIAS BIBLIOGRÁFICAS}

Achten, J., \& Jeukendrup, A. E. (2003). Heart rate monitoring. Sports Medicine, 33(7), 517538. https://doi.org/10.2165/00007256-200333070-00004

Billman, G. E. (2015). The LF/HF ratio does not accurately measure cardiac sympatho-vagal balance. Heart Rate Variability: Clinical Applications and Interaction between HRV and Heart Rate, 54.

Boron, W. F., \& Boulpaep, E. L. (2012). Medical Physiology, 2e Updated Edition: with STUDENT CONSULT Online Access: Elsevier Health Sciences.

Borresen, J., \& Lambert, M. I. (2008). Autonomic control of heart rate during and after exercise. Sports Medicine, 38(8), 633-646. https://doi.org/10.2165/00007256200838080-00002

Cardiology, T. F. o. t. E. S. o. (1996). Heart rate variability standards of measurement, physiological interpretation, and clinical use. Eur Heart J, 17, 354-381. https://doi.org/10.1093/oxfordjournals.eurheartj.a014868

Casadei, B., Cochrane, S., JOHNSOTON, J., Conway, J., \& Sleight, P. (1995). Pitfalls in the interpretation of spectral analysis of the heart rate variability during exercise in humans. Acta Physiologica Scandinavica, 153(2), 125-131. https://doi.org/10.1111/j.1748-1716.1995.tb09843.x

Cherniack, N., Adams, E., Prabhakar, N., Haxhiu, M., \& Mitra, J. (1989). Integration of cardiorespiratory responses in the ventrolateral medulla. Progress in brain research, 81, 215-220. https://doi.org/10.1016/S0079-6123(08)62011-7

Coleridge, H. M., \& Coleridge, J. C. G. (1986). Reflexes evoked from tracheobronchial tree and lungs. In A. P. Fishman (Ed.), Handbook of physiology. Section 3: The Respiratory System (Vol. II: Control of breathing, part I, pp. 407-413). Bethesda, Maryland: American Physiological Society. 
Cottin, F., Durbin, F., \& Papelier, Y. (2004). Heart rate variability during cycloergometric exercise or judo wrestling eliciting the same heart rate level. European journal of applied physiology, 91(2-3), 177-184. https://doi.org/10.1007/s00421-003-0969-1

Cottin, F., Papelier, Y., \& Escourrou, P. (1999). Effects of exercise load and breathing frequency on heart rate and blood pressure variability during dynamic exercise. International journal of sports medicine, 20(4), 232-238. https://doi.org/10.1055/s2007-971123

Das, G. (1989). Therapeutic review. Cardiac effects of atropine in man: an update. International journal of clinical pharmacology, therapy, and toxicology, 27(10), 473477.

Evans, J. M., Ziegler, M. G., Patwardhan, A. R., Ott, J. B., Kim, C. S., Leonelli, F. M., \& Knapp, C. F. (2001). Gender differences in autonomic cardiovascular regulation: spectral, hormonal, and hemodynamic indexes. Journal of Applied Physiology, 91(6), 2611-2618. https://doi.org/10.1152/jappl.2001.91.6.2611

Goldberger, J. J., Challapalli, S., Tung, R., Parker, M. A., \& Kadish, A. H. (2001). Relationship of heart rate variability to parasympathetic effect. Circulation, 103(15), 1977-1983. https://doi.org/10.1161/01.CIR.103.15.1977

Hagerman, I., Berglund, M., Lorin, M., Nowak, J., \& Sylvén, C. (1996). Chaos-related deterministic regulation of heart rate variability in time-and frequency domains: effects of autonomic blockade and exercise. Cardiovascular research, 31(3), 410418. https://doi.org/10.1016/S0008-6363(95)00084-4

Hughson, R. L., Sutton, J. R., Fitzgerald, J. D., \& Jones, N. L. (1977). Reduction of intrinsic sinoatrial frequency and norepinephrine response of the exercised rat. Canadian journal of physiology and pharmacology, 55(4), 813-820. https://doi.org/10.1139/y77-109

Jewett, D. (1964). Activity of single efferent fibres in the cervical vagus nerve of the dog, with special reference to possible cardio-inhibitory fibres. The Journal of Physiology, 175(3), 321. https://doi.org/10.1113/jphysiol.1964.sp007520

Jindal, V., Gupta, S., \& Das, R. (2013). Molecular mechanisms of meditation. Molecular neurobiology, 48(3), 808-811. https://doi.org/10.1007/s12035-013-8468-9

Katona, P. G., \& Jih, F. (1975). Respiratory sinus arrhythmia: noninvasive measure of parasympathetic cardiac control. J Appl Physiol, 39(5), 801-805. https://doi.org/10.1152/jappl.1975.39.5.801

Korner, P. (1971). The central nervous system and physiological mechanisms of "Optimal" cardiovascular control. Amer. J. exp. Biol. Med. Sci, 49, 319-343. https://doi.org/10.1038/icb.1971.35

Korner, P. I. (1971). Integrative neural cardiovascular control. Physiological Reviews, 51(2), 312-367. https://doi.org/10.1152/physrev.1971.51.2.312

Kunze, D. L. (1972). Reflex discharge patterns of cardiac vagal efferent fibres. The Journal of Physiology, 222(1), 1. https://doi.org/10.1113/jphysiol.1972.sp009784

Kuo, C.-D., Chen, G.-Y., Lai, S.-T., Wang, Y.-Y., Shih, C.-C., \& Wang, J.-H. (1999). Sequential changes in heart rate variability after coronary artery bypass grafting. The American journal of cardiology, 83(5), 776-779. https://doi.org/10.1016/S00029149(98)00989-8

Kuo, T. B., Lin, T., Yang, C. C., Li, C.-L., Chen, C.-F., \& Chou, P. (1999). Effect of aging on gender differences in neural control of heart rate. American Journal of PhysiologyHeart and Circulatory Physiology, 277(6), H2233-H2239. https://doi.org/10.1152/ajpheart.1999.277.6.H2233 
Lombardi, F. (2000). Chaos theory, heart rate variability, and arrhythmic mortality. Circulation, 101(1), 8-10. https://doi.org/10.1161/01.CIR.101.1.8

Ludwig, C. (1847). Beitrage zur Kenntniss des Einflusses der Respirationsbewegungen auf den Blutlauf im Aortensysteme. Arch. Anat. Physiol, 13, 242-302.

Malmo, R. B., Shagass, C., Davis, J., Cleghorn, R., Graham, B., \& Goodman, A. J. (1948). Standardized pain stimulation as controlled stress in physiological studies of psychoneurosis. Science, $108(2810)$, 509-511. https://doi.org/10.1126/science.108.2810.509

Mourot, L., Bouhaddi, M., Perrey, S., Cappelle, S., Henriet, M. T., Wolf, J. P., . . Regnard, J. (2004). Decrease in heart rate variability with overtraining: assessment by the Poincare plot analysis. Clinical physiology and functional imaging, 24(1), 10-18. https://doi.org/10.1046/j.1475-0961.2003.00523.x

Pagani, M., Lombardi, F., Guzzetti, S., Rimoldi, O., Furlan, R., Pizzinelli, P., . . . Piccaluga, E. (1986). Power spectral analysis of heart rate and arterial pressure variabilities as a marker of sympatho-vagal interaction in man and conscious dog. Circulation research, 59(2), 178-193. https://doi.org/10.1161/01.RES.59.2.178

Piccirillo, G., Ogawa, M., Song, J., Chong, V. J., Joung, B., Han, S., . . Chen, P.-S. (2009). Power spectral analysis of heart rate variability and autonomic nervous system activity measured directly in healthy dogs and dogs with tachycardia-induced heart failure. Heart Rhythm, 6(4), 546-552. https://doi.org/10.1016/j.hrthm.2009.01.006

Rosenblueth, A., \& Simeone, F. (1934). The interrelations of vagal and accelerator effects on the cardiac rate. American Journal of Physiology--Legacy Content, 110(1), 42-55. https://doi.org/10.1152/ajplegacy.1934.110.1.42

Sandercock, G., \& Brodie, D. (2006). The use of heart rate variability measures to assess autonomic control during exercise. Scandinavian journal of medicine \& science in sports, 16(5), 302-313. https://doi.org/10.1111/j.1600-0838.2006.00556.x

Sandercock, G., Bromley, P. D., \& Brodie, D. A. (2005). Effects of exercise on heart rate variability: inferences from meta-analysis. Med Sci Sports Exerc, 37(3), 433-439. https://doi.org/10.1249/01.MSS.0000155388.39002.9D

Sarmiento Montesdeoca, S., García-Manso, J. M., Martín-González, J., Medina, G., Calderón, F., \& Rodríguez Ruíz, D. (2009). Análisis tiempo-frecuencia de la variabilidad de la frecuencia cardiaca (VFC) durante la aplicación de un esfuerzo incremental en ciclistas. $d E s d E$ la ExpEriEncia, 71.

Schramm, L. P. (2006). Spinal sympathetic interneurons: their identification and roles after spinal cord injury. Progress in brain research, 152, 27-37. https://doi.org/10.1016/S0079-6123(05)52002-8

Silva, C., Pereira, L. M., Cardoso, J. R., Moore, J. P., \& Nakamura, F. Y. (2014). The Effect of physical training on heart rate variability in healthy children: A systematic review with meta-analysis. Pediatr Exerc Sci, 26(2), 147-158. https://doi.org/10.1123/pes.2013-0063

Silva, V. P., Oliveira, N. A., Silveira, H., Mello, R. G. T., \& Deslandes, A. C. (2015). Heart rate variability indexes as a marker of chronic adaptation in athletes: a systematic review. Annals of Noninvasive Electrocardiology, 20(2), 108-118. https://doi.org/10.1111/anec.12237

Singh, J. P., Larson, M. G., O'Donnell, C. J., \& Levy, D. (2001). Genetic factors contribute to the variance in frequency domain measures of heart rate variability. Autonomic Neuroscience, 90(1), 122-126. https://doi.org/10.1016/S1566-0702(01)00277-6 
Singh, J. P., Larson, M. G., O’Donnell, C. J., Tsuji, H., Corey, D., \& Levy, D. (2002). Genome scan linkage results for heart rate variability (the Framingham Heart Study). The American journal of cardiology, 90(12), 1290-1293. https://doi.org/10.1016/S00029149(02)02865-5

Singh, J. P., Larson, M. G., O’Donnell, C. J., Tsuji, H., Evans, J. C., \& Levy, D. (1999). Heritability of Heart Rate Variability The Framingham Heart Study. Circulation, 99(17), 2251-2254. https://doi.org/10.1161/01.CIR.99.17.2251

Tanaka, H., Dinenno, F. A., Monahan, K. D., Clevenger, C. M., DeSouza, C. A., \& Seals, D. R. (2000). Aging, habitual exercise, and dynamic arterial compliance. Circulation, 102(11), 1270-1275. https://doi.org/10.1161/01.CIR.102.11.1270

Taylor, E. W., Leite, C. A., Sartori, M. R., Wang, T., Abe, A. S., \& Crossley, D. A. (2014). The phylogeny and ontogeny of autonomic control of the heart and cardiorespiratory interactions in vertebrates. The Journal of experimental biology, 217(5), 690-703. https://doi.org/10.1242/jeb.086199

Tsuji, H., Venditti, F. J., Manders, E. S., Evans, J. C., Larson, M. G., Feldman, C. L., \& Levy, D. (1996). Determinants of heart rate variability. Journal of the American College of Cardiology, 28(6), 1539-1546. https://doi.org/10.1016/S0735-1097(96)00342-7

Turner, D. L. (1991). Cardiovascular and respiratory control mechanisms during exercise: an integrated view. J Exp Biol, 160, 309-340.

Umetani, K., Singer, D. H., McCraty, R., \& Atkinson, M. (1998). Twenty-four hour time domain heart rate variability and heart rate: relations to age and gender over nine decades. Journal of the American College of Cardiology, 31(3), 593-601. https://doi.org/10.1016/S0735-1097(97)00554-8

Verberne, A. J., \& Owens, N. C. (1998). Cortical Modulation of theCardiovascular System. Progress in neurobiology, 54(2), 149-168. https://doi.org/10.1016/S03010082(97)00056-7

\footnotetext{
Número de citas totales / Total references: 45 (100\%)

Número de citas propias de la revista / Journal's own references: $0(0 \%)$
}

Rev.int.med.cienc.act.fís.deporte - vol. 20 - número 78 - ISSN: 1577-0354 